\title{
Facial Rejuvenating Effects of Mesotherapy Using a New Polycomponent Formulation
}

\author{
Gyu Sik Jung ${ }^{1}$ \\ Jeung Ryeol Eom ${ }^{1}$ \\ Hyeon Jun Jeon ${ }^{1}$ \\ Seung Gyun $\mathrm{Chu}^{1}$ \\ Eugene $\mathrm{Oh}^{2}$ \\ Jae Woong Lee ${ }^{3}$
}

\footnotetext{
${ }^{1}$ Department of Plastic and Reconstructive Surgery, School of Medicine, Kyungpook National University, Daegu, Korea

${ }^{2}$ Department of Chemical Engineering, Pohang University of Science and Technology (POSTECH), Pohang, Korea

${ }^{3}$ Giho Cultural Heritage Research Center,

Anseong, Korea
}

Received July 28, 2018

Accepted August 20, 2018

\author{
Correspondence \\ Gyu Sik Jung \\ Department of Plastic and Reconstructive \\ Surgery, School of Medicine, Kyungpook National \\ University, 130 Dongdeok-ro, Jung-gu, Daegu \\ 41944, Korea \\ Tel.: +82-53-200-5685 \\ Fax: +82-53-425-3879 \\ E-mail: jksheartanaver.com \\ (c) Korean Society for Laser Medicine and Surgery \\ (c) This is an open access article distributed under the \\ terms of the Creative Commons Attribution Non- \\ Commercial License (http://creativecommons.org/ \\ licenses/by-nc/4.0) which permits unrestricted non- \\ commercial use, distribution, and reproduction in any \\ medium, provided the original work is properly cited.
}

\begin{abstract}
Background and Objectives
Facial rejuvenation via mesotherapy using a new polycomponent formulation was employed to improve skin texture. In this study, we evaluated the rejuvenating effects of a new polycomponent formulation through mesotherapy using a microneedle therapy system (MTS).

\section{Materials and Methods}

Twenty patients underwent a series of facial procedures, including three sessions performed at 2-week intervals and two sessions at 4-week intervals. Subsequently, $3 \mathrm{~mL}$ of new polycomponent formulation was applied to the entire face and MTS was used. Patient satisfaction scoring was conducted by three plastic surgeons who evaluated the improvement of fine wrinkles, skin tightness, hydration, and sweat pore size. In addition, photographs of the patients were taken at each visit and 2 months after the last treatment session.
\end{abstract}

\section{Results}

The patients were satisfied with the therapeutic outcomes. The scores of the surgeons for the improvement of fine wrinkles, skin tightness, hydration, and sweat pore size were high.

\section{Conclusion}

Minimally invasive mesotherapy using a new polycomponent formulation can improve the clinical appearance of facial skin. However, a largerscale and well-controlled investigation of the clinical efficacy and safety of this technique is needed to confirm these findings.

\section{Key words}

Mesotherapy; Microneedle therapy system; Facial rejuvenation; Aging; Fibroblasts 


\section{INTRODUCTION}

Aging, especially skin aging, has received increasing attention in modern society. Facial aging is a complex process with two important components: facial volume loss and repeated muscle movements that create wrinkles. Fibroblasts are the major dermal cells that produce and maintain the extracellular connective tissues that are crucial for maintaining skin youth. Functional impairment or reduced biosynthetic activity of fibroblasts (fibroblast collapse), which is caused by the loss of physical interactions between fibroblasts and the surrounding extracellular matrix, reflects as skin aging. ${ }^{1-3}$ Aged fibroblasts cannot produce the extracellular matrix. In addition, the number of fibroblasts decreases with age; subsequently, the amount of hyaluronic acid and collagen produced by fibroblasts and the number of cells that comprise the extracellular matrix decrease. ${ }^{4}$ The secretion of enzymes that degrade collagen also increases.

With the emergence of anti-aging theories, emphasis on mesotherapy has increased. The term mesotherapy is derived from the Greek words "mesos" (middle) and "therapeia" (to treat medically). It was first introduced by Pistor in $1958 .{ }^{5}$ The goal of mesotherapy is to delay and improve the skin changes caused by aging. ${ }^{6}$ It is a minimally invasive procedure that involves simultaneous injection of small amounts of hyaluronic acids, vitamins, antioxidants, enzymes, and humectants into the subcutaneous layer to stimulate the biosynthetic ability of fibroblasts and to provide an optimal physiologic environment for fibroblasts to increase collagen and elastin syntheses and supply basic active molecules. ${ }^{7.8}$ It is one of the newest techniques in cosmetic medicine proposed to rejuvenate aging skin and can be performed by medical and non-medical professionals. The desired final effect is a firm, bright, and moisturized skin obtained by the injection of suitable products, which are biocompatible and absorbable by the superficial dermis. These products or molecules used in mesotherapy hydrate and activate fibroblasts to promote skin rejuvenation. To date, only a few studies have reported the facial rejuvenating effects of polycomponent mesotherapy.?

The a new polycomponent formulation, an example of a functional polycomponent formulation, contains vitamins, minerals, amino acids, nucleic acids, coenzymes, antioxidants, and hyaluronic acid. The new polycomponent formulation has been used to improve facial wrinkles. Previously, concerns existed about improving deep wrinkles and volume deficiency; however, recently, attention has shifted to improving skin texture. To address this new in- terest, this study aimed to describe improvements in skin texture using mesotherapy with a new polycomponent formulation.

\section{MATERIALS AND METHODS}

Twenty female patients, aged between 25 to 54 years, with dry and aging facial skin were enrolled in the study. All patients provided written informed consent. Patients were excluded if they were pregnant or breastfeeding and had an autoimmune disease, skin cancer, hypersensitivity to hyaluronic acid, and active skin diseases such as an infection, eczema, and dermatitis. In addition, patients who had been treated with other skin rejuvenation procedures, such as peeling, botulinum toxin, filler, or laser therapy, and had undergone facial surgery within the previous 3 months were excluded.

The procedure was as follows. First, the face was washed and an anesthetic cream was applied. The face was then disinfected using chlorhexidine after $30 \mathrm{~min}$. Thereafter, $2 \mathrm{~mL}$ of a new polycomponent formulation (NCTF 135HA ${ }^{\circledR}$; Filorga, Paris, France) was applied to the entire face and a microneedle therapy system (MTS) was used to scan the face six to eight times. Subsequently, $1 \mathrm{~mL}$ of a new polycomponent formulation was applied to the face and was allowed to absorb. Next, a healing cream was applied, and the face was disinfected with chlorhexidine. No additional procedures were performed simultaneously. To minimize the risk of complications, such as hematoma, the needling site was gently compressed and cooled with an ice pack for several minutes after the procedure. A photograph of the patient's face was taken before each treatment session and 2 months after the last treatment session. Patients were also asked about their opinions regarding aesthetic satisfaction using a questionnaire survey after 2 months of the last session. The degree of satisfaction was evaluated using points (point 1, very unsatisfied; point 2, unsatisfied; point 3, okay; point 4, satisfied; point 5, very satisfied).

Three plastic surgeons provided scores for each patient according to a 5-point scale in four categories: improvement of micro-wrinkle, skin tightness, hydration, and sweat pore size. The collected data were statistically measured and presented as mean and standard deviation (SD).

\section{RESULTS}

All 20 patients completed the treatment sessions. The mean age of the patients was 43.7 years. No major com- 
plications, such as infection and hematoma, occurred during the course of the treatment. One patient experienced mild erythema after the treatment, which subsided 3 days later and healed without any sequelae. Two patients had transient, mild bruises after the treatment (Fig. 1-3).

Patient satisfaction scores were 4.6 points (SD, 0.42) overall, 4.5 points (SD, 0.32) for fine wrinkle, 4.2 points (SD, 0.52 ) for skin tightness, 4.7 points (SD, 0.32) for hydration, and 4.8 points (SD, 0.64) for sweat pore size. Surgeon satisfaction scores were 4.7 points (SD, 0.54) overall, 4.7 points (SD, 0.55) for fine wrinkle, 4.3 points (SD, 0.54) for skin tightness, 4.8 points (SD, 0.44) for hydration, and 4.9 points (SD, 0.32) for sweat pore size (Fig. 4).

\section{DISCUSSION}

The new polycomponent formulation contains 53 dif- ferent compounds, including vitamins, amino acids, minerals, coenzymes, nucleic acids, and hyaluronic acids (Table 1). Therefore, its use must be based on a thorough understanding of the roles each compound plays in biochemical processes related to the skin. Mesotherapy using MTS for soft facial tissues is reported to be safe. Mild pain, redness, swelling, and bruising are symptoms that resolve quickly and can be expected after mesotherapy using MTS. ${ }^{10}$ Mesotherapy most commonly involves multiple injections of bioactive substances for facial contouring through lipolysis. However, microneedling utilizes controlled needle penetration of the skin to stimulate the wound healing cascade and induce regeneration of skin components. There is a difference between mesotherapy and microneedling but they can be considered as similar concepts. $^{2}$

The new polycomponent formulation contains a combination of 53 polycompounds, including 23 amino acids,
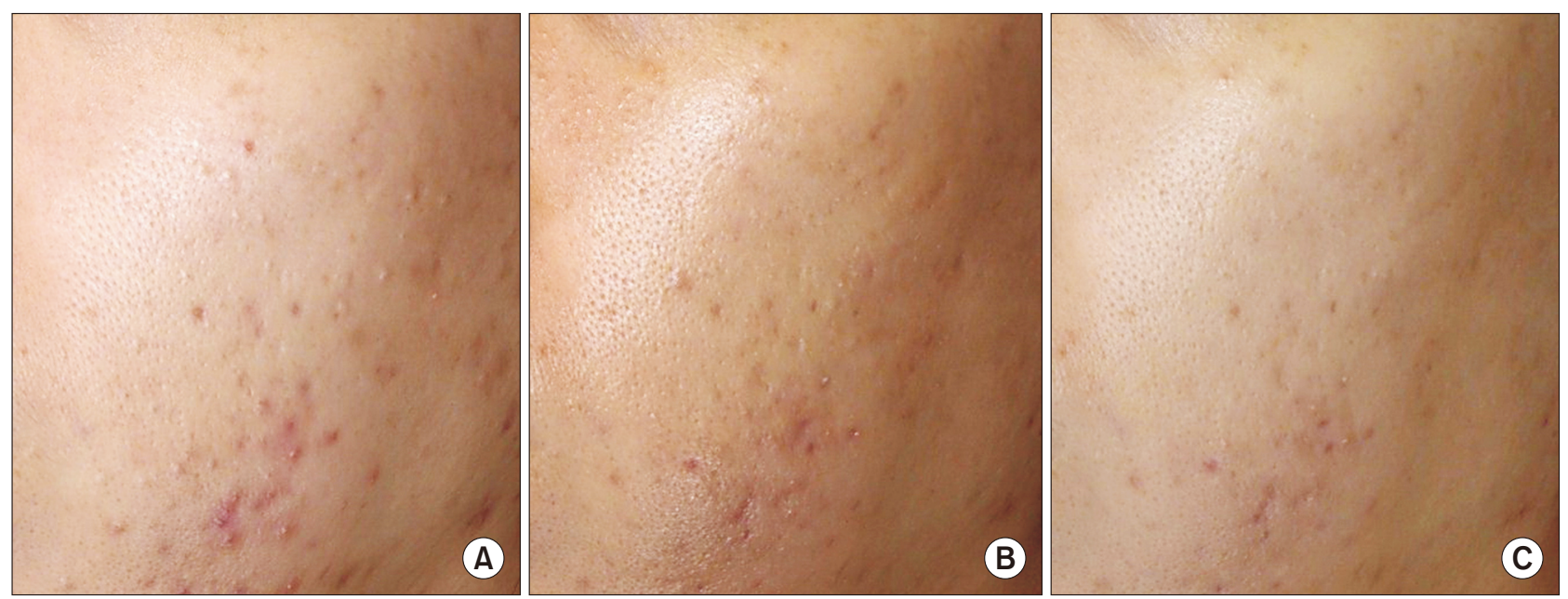

Fig. 1. Photographs of a 27-year-old woman. (A) Preoperative image. (B) After three sessions. (C) After all five treatment sessions.

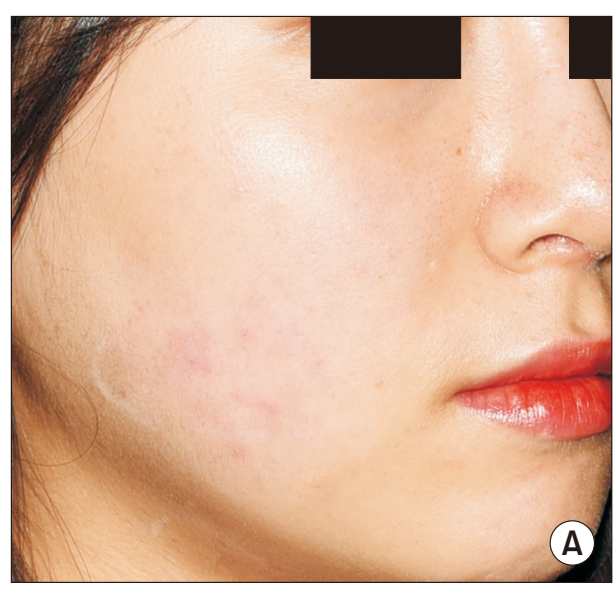

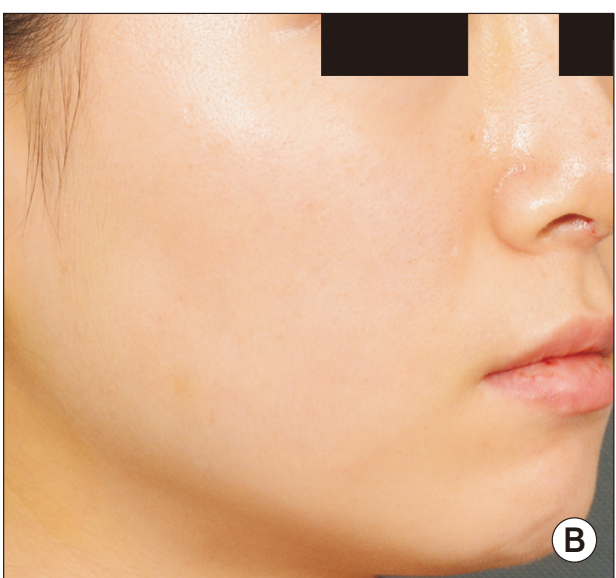

Fig. 2. Photographs of a 32-year-old woman. (A) Preoperative photograph. (B) Photograph after all five treatment sessions. 
Table 1. The new polycomponent formulation

\begin{tabular}{lc}
\hline \multicolumn{1}{c}{ Class } & Components \\
\hline Vitamins & Vitamin A (retinol), vitamin C (ascorbic acid), vitamin B1 (thiamine), Vitamin B2 (riboflavin), vitamin B3 (nicotinamide), \\
& vitamin B5 (pantothenic acid), vitamin B6 (pyridoxin), vitamin B8 (biotin), vitamin B9 (folic acid), vitamin B10 (p- \\
aminobenzoic acid), vitamin B12 (cyanocobalamin) & Calcium chloride, potassium chloride, magnesium, sulfate, sodium acetate, sodium chloride, sodium, dihydrogen \\
phosphate & Alanine, asparagine, arginine, aspartic acid, cystine, glutamine, glutamic acid, glycine, histidine, hydroxyproline, \\
Amino acids & isoleucine, leucine, lysine, methionine, ornithine, phenylalanine, proline, serine, taurine, threonine, tryptophan, \\
& tyrosine, valine \\
Deoxyadenosine, deoxycytidine, deoxyguanosine, deoxythymidine, methylcytosine, thiamine pyrophosphate, \\
pounds \\
coenzyme A, flavin adenine dinucleotide, nicotinamide adenine dinucleotide, nicotinamide adenine dinucleotide \\
phosphate, uridine-5'-triphosphate, glutathione, non-reticulated, sodium hyaluronate
\end{tabular}

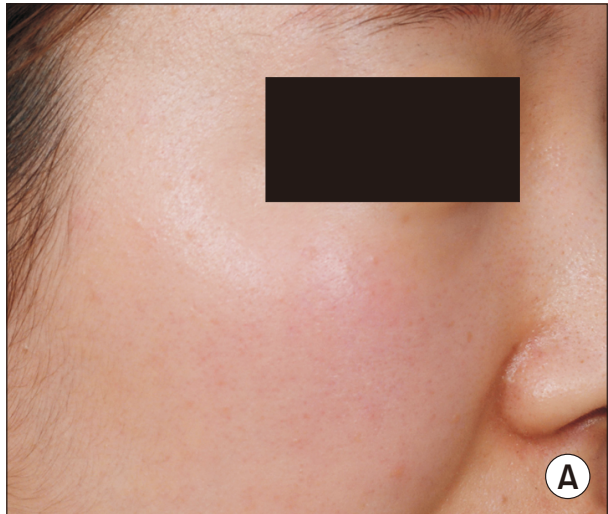

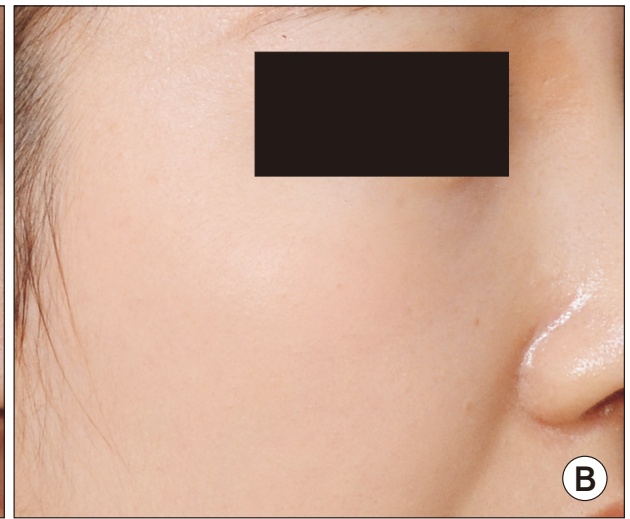

Fig. 3. Photographs of a 33-year-old woman. (A) Preoperative photograph. (B) Photograph after all five treatment sessions.
6 coenzymes, 5 nucleic acids, antioxidants, 12 vitamins, hyaluronic acids, and 6 minerals, and creates an optimal environment for restoring the function of fibroblasts that play crucial roles in skin aging. Vitamins play an important role related to skin aging. Retinol, vitamin A has an antioxidant effect and controls skin regeneration, melanocyte, and sebaceous gland activity. Vitamin B is involved in many cell regulatory processes and acts as a coenzyme. Thiamine, vitamin B1 produces energy from carbohydrates and derives ribose and deoxyribose from glucose. Riboflavin, vitamin B2 delivers energy from carbohydrates and fat and is involved in the cellular redox metabolism. Ascorbic acid, vitamin $\mathrm{C}$ is an important antioxidant that accelerates deoxyribonucleic acid synthesis and is essential for collagen synthesis. Tocopherol, vitamin $E$ is an important compound with high levels of antioxidative activity. Inositol is a vitamin-like active substance that acts as a signaling molecule that regulates important markers related to cellular homeostasis, such as intracellular calcium levels, and contributes to the maintenance of cell membrane capacitance.

Calcium is an ion that controls cellular homeostasis

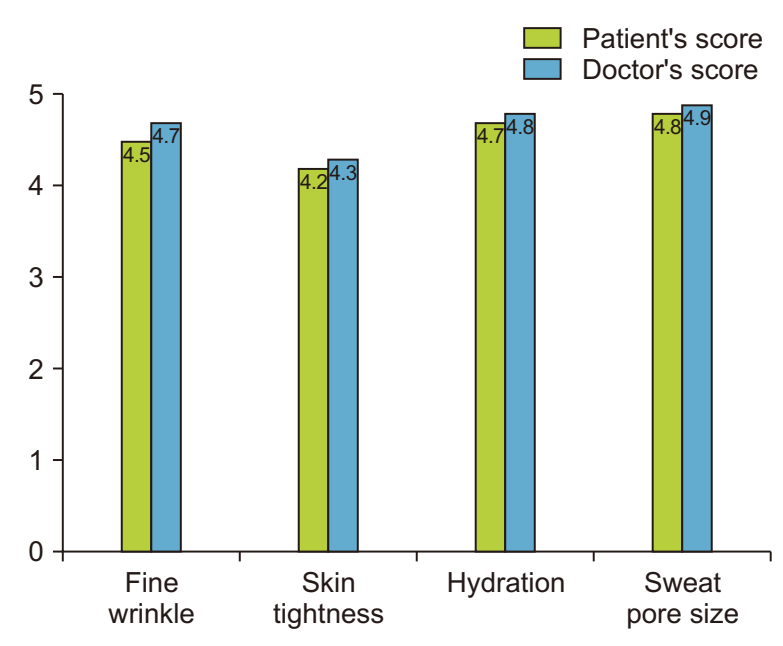

Fig. 4. Patients' and surgeons' satisfaction scores. Both patients and surgeons show high levels of satisfaction. The surgeons are more satisfied than the patients.

and is an essential component of cell walls and other biological membranes. Magnesium is needed to maintain a large number of normal enzymatic reactions. Nucleo- 
sides are needed during protein synthesis to copy DNA in preparation for fibroblast division and ribonucleic acid production. Coenzymes are catalysts of biochemical reactions. Hyaluronic acids can store and maintain water up to 1000 times their weights and maintain skin moisture. In addition, they have antibacterial, antifungal, and antioxidant characteristics and promote de novo collagen synthesis. ${ }^{11-13}$

In conclusion, minimally invasive mesotherapy with a new polycomponent formulation can improve the clinical appearance of the skin by maintenance and/or restoration of a healthy and youthful skin texture. However, a largerscale and well-controlled study on the clinical efficacy and safety of this technique is needed to confirm this finding.

\section{CONFLICT OF INTEREST}

None of the authors has commercial associations or financial disclosures that might create a conflict of interest regarding the methods or results presented in this article.

\section{REFERENCES}

1. Rohrich RJ. Mesotherapy: what is it? Does it work? Plast Reconstr Surg 2005;115:1425.

2. Pistor M. What is mesotherapy? Chir Dent Fr 1976;46:59-60.

3. El-Komy M, Hassan A, Tawdy A, Solimon M, Hady MA. Hair loss at injection sites of mesotherapy for alopecia. J Cosmet Dermatol 2017;16:28-30.

4. Lee JC, Daniels MA, Roth MZ. Mesotherapy, microneedling, and chemical peels. Clin Plast Surg 2016;43:583-95.
5. Ahmed NA, Mohammed SS, Fatani MI. Treatment of periorbital dark circles: comparative study of carboxy therapy vs chemical peeling vs mesotherapy. J Cosmet Dermatol 2018; in press.

6. Balevi A, Ustuner P, Özdemir M. Salicylic acid peeling combined with vitamin c mesotherapy versus salicylic acid peeling alone in the treatment of mixed type melasma: a comparative study. J Cosmet Laser Ther 2017;19:294-9.

7. Tedeschi A, Lacarrubba F, Micali G. Mesotherapy with an intradermal hyaluronic acid formulation for skin rejuvenation: an intrapatient, placebo-controlled, long-term trial using highfrequency ultrasound. Aesthetic Plast Surg 2015;39:129-33.

8. Amin SP, Phelps RG, Goldberg DJ. Mesotherapy for facial skin rejuvenation: a clinical, histological, and electron microscopic evaluation. Dermatol Surg 2006;32:1467-72.

9. Savoia A, Landi S, Baldi A. A new minimally invasive mesotherapy technique for facial rejuvenation. Dermatol Ther (Heidelb) 2013;3:83-93.

10. El-Domyati M, El-Ammawi TS, Moawad O, El-Fakahany $H$, Medhat W, Mahoney MG, et al. Efficacy of mesotherapy in facial rejuvenation: a histological and immunohistochemical evaluation. Int J Dermatol 2012;51:913-9.

11. Deglesne PA, Arroyo R, Ranneva E, Deprez P. In vitro study of RRS HA injectable mesotherapy/biorevitalization product on human skin fibroblasts and its clinical utilization. Clin Cosmet Investig Dermatol 2016;9:41-53.

12. Herreros FO, Moraes AM, Velho PE. Mesotherapy: a bibliographical review. An Bras Dermatol 2011;86:96-101.

13. Prikhnenko S. Polycomponent mesotherapy formulations for the treatment of skin aging and improvement of skin quality. Clin Cosmet Investig Dermatol 2015;8:151-7. 